\title{
Future Directions for Video Fire Detection
}

\author{
STEVEN VERSTOCKT ${ }^{1,2}$, NELE TILLEY ${ }^{3}$, BART MERCI ${ }^{3}$, CHARLES HOLLEMEERSCH ${ }^{1}$, BART \\ SETTE $^{4}$, SOFIE VAN HOECKE ${ }^{2}$, PETER LAMBERT ${ }^{1}$, and RIK VAN DE WALLE ${ }^{1}$ \\ ${ }^{1}$ Department of Electronics and Information Systems \\ Multimedia Lab, Ghent University - IBBT \\ Gaston Crommenlaan 8, bus 201, B-9050 Ledeberg-Ghent, Belgium \\ ${ }^{2}$ Electronics and Information Technology Lab \\ University College West Flanders, \\ Ghent University Association, Graaf Karel de Goedelaan 5, 8500 Kortrijk, Belgium \\ ${ }^{3}$ Department of Flow, Heat and Combustion Mechanics \\ Ghent University, \\ Sint-Pietersnieuwstraat 41, B-9000 Ghent, Belgium \\ ${ }^{4}$ Warringtonfiregent (WFRGent NV) \\ Ottergemsesteenweg 711, B-9000 Ghent, Belgium
}

\begin{abstract}
To accomplish more valuable and more accurate video fire detection, this paper points out future directions and discusses first steps which are now being taken to improve the vision-based detection of smoke and flames. First, an overview is given of the state-of-the-art detection methods in the visible and infrared spectral range. Then, a novel multi-sensor smoke and flame detector is proposed which combines the multimodal information of low-cost visual and thermal infrared detection results. Experiments on fire and nonfire multi-sensor sequences indicate that the combined detector yields more accurate results, with fewer false alarms, than either detector alone. Next, a framework for multi-view fire analysis is discussed to overcome the lack in a video-based fire analysis tool and to detect valuable fire characteristics at the early stage of the fire. As prior experimental results show, this combined analysis from different viewpoints provides more valuable fire characteristics. Information about 3-D fire location, size and growth rate can be extracted from the video data in practically no time. Finally, directions towards standardized evaluation and video-driven fire forecasting are suggested.
\end{abstract}

KEYWORDS: detection, multi-view, multi-sensor, video, modeling.

\section{INTRODUCTION}

Current research [1] shows that video-based fire detection promises fast detection and can be a viable alternative for the more traditional techniques. However, due to the variability of shape, motion, transparency, colors, and patterns of smoke and flames, existing approaches are still vulnerable to false alarms. On the other hand, video-based fire alarm systems mostly only detect the presence of fire. To understand the fire, however, detection is not enough. Effective response to fire requires accurate and timely information of its evolution. As an answer to both problems a multi-sensor fire detector and a multiview fire analysis framework [2] is proposed, which can be seen as the first steps towards more valuable and accurate video fire detection (VFD).

Although different sensors can be used for multi-sensor fire detection, we believe that the added value of IR cameras in the long wave infra-red range (LWIR) will be the highest. Various facts support this idea. First of all, existing VFD algorithms have inherent limitations, such as the need for sufficient and specific lighting conditions. Thermal IR imaging sensors image emitted light, not reflected light, and do not have this limitation. Also, the further one goes in the IR spectrum the more the visual perceptibility decreases and the thermal perceptibility increases. As such, hot objects will be best visible and less disturbed by other objects in the LWIR spectral range. By combining the thermal and visual characteristics of moving objects in registered LWIR, as well as visual images, more robust fire detection can be achieved. Since visual misdetections can be corrected by LWIR detections and vice versa, fewer false alarms will occur.

Due to the transparency of smoke in LWIR images, its absence can be used to distinguish between smoke and smoke-like moving objects. Since ordinary moving objects, such as people and cars, produce similar silhouettes in background-subtracted visual and thermal IR images, the coverage between these images is quasi constant. Smoke, contrarily, will only be detected in the visual images, and as such the coverage will 
start to decrease. Due to the dynamic character of the smoke, this decrease will also show a high degree of disorder. By focusing on both coverage behaviors, smoke can be detected. On the basis of all these facts, the use of LWIR in combination with ordinary VFD is considered to be a win-win. This is also confirmed by experiments presented in this paper, in which the fused detectors perform better than either sensor alone.

In order to actually understand and interpret the fire, however, detection is not enough. It is also important to have a clear understanding of the fire development and the location. Where did the fire start? What is the size of the fire? What is the direction of smoke propagation? How is the fire growing? The answer to each of these questions plays an important role in safety analysis and fire fighting/mitigation, and is essential in assessing the risk of escalation. Nevertheless, the majority of the detectors that are currently in use just ring the bell, i.e., they only detect the presence of fire, and are not able to model fire evolution. In order to accomplish more valuable fire analysis, a framework for video fire analysis has become one of the main goals of our work. By fusing video fire detection results of multiple cameras using the framework, valuable fire characteristics can be detected at the early stage of the fire. The framework merges the single-view detection results of the multiple cameras by homographic projection onto multiple horizontal and vertical planes, which slice the scene. The crossings of these slices create a 3-D grid of virtual sensor points. Using this grid and subsequent spatial and temporal 3-D clean-up filters, information about the location of the fire, its size and its propagation can be instantly extracted from the video data.

Important to mention is that interviews with partners in the field revealed that for our specific 'use case', i.e., the detection of fire in public places (such as shopping malls and airports), it is commercially viable to use multiple cameras instead of traditional point, beam, or aspirating type fire/smoke detectors. Most of the time these cameras are already available and used for other purposes, such as ordinary video surveillance. As such, mostly no additional hardware cost is needed. Furthermore, increasing the number of cameras does not much affect the software/processing costs, since the detections itself can run in parallel. By combining the detection results, valuable characteristics about fire spread etc. can be obtained with low cost, which cannot be achieved with the more 'traditional' detectors.

\section{VIDEO FIRE DETECTION}

First, an overview is given of state-of-the-art VFD algorithms in visible light with a particular focus on the underlying features. Next, the (limited) work in IR-based fire detection is presented and different techniques are highlighted which can be of use in LWIR fire detection.

\section{VFD in Visible Light}

The several vision-based fire and smoke detection algorithms that have been proposed in the literature have led to a large amount of VFD algorithms that can be used to detect the presence of fire at an early stage. Each of these algorithms detects flames or smoke by analyzing one or more fire features in visible light [1].

Color was one of the first features used in VFD and is still by far the most popular [3]. The majority of the color-based approaches in VFD makes use of RGB color space, sometimes in combination with the saturation of HSI (hue-saturation-intensity) color space [4,5]. The main reason for using RGB is the equality in RGB values of smoke pixels and the easily distinguishable red-yellow range of flames. Although the test results in the referenced work seems promising at first, the variability in color, density, lighting, and background do raise questions about the applicability of RGB in real world detection systems. In Ref. [1], the authors discuss the detection of chrominance decrease as a superior method.

Other frequently used fire features are flickering [5,6] and energy variation $[1,7,8]$. Both focus on the temporal behavior of flames and smoke. Flickering refers to the temporal intermittency with which pixels appear and disappear at the edges of turbulent flames. Energy variation refers to the temporal disorder of pixels in the high-pass components of the discrete wavelet transformed images of the camera. Fire also has the unique characteristic that it does not remain a steady color, i.e., the flames are composed of several varying colors within a small area. Spatial difference analysis [5,9] focuses on this feature and analyses the spatial color variations in pixel values to eliminate ordinary fire-colored objects with a solid flame color. Also an interesting feature for fire detection is the disorder of smoke and flame regions over time. Some examples of frequently used metrics to measure this disorder are randomness of area size [10], boundary roughness [8], and turbulence variance [11]. 
Based on the analysis of our own experiments [12] and the discussed state-of-the-art, a low-cost flame detector is presented in (Fig. 1). The detector starts with a dynamic background subtraction [1,9], which extracts moving objects by subtracting the video frames with everything in the scene that remains constant over time, i.e. the estimated background. To avoid unnecessary computational work and to decrease the number of false alarms caused by noisy objects, a morphological opening, which filters out the noise, is performed after the dynamic background subtraction. Each of the remaining foreground (FG) objects in the video images is then further analyzed using a set of visual flame features. In the case of a fire object, the selected features, i.e. spatial flame color disorder, principal orientation disorder and bounding box disorder, vary considerably over time. Due to this high degree of disorder, extrema analysis is chosen as a technique to easily distinguish between flames and other objects. It is related to the number of extremes, i.e., local maxima and minima, in the set of data points. For more detailed information the reader is referred to Ref. [12].

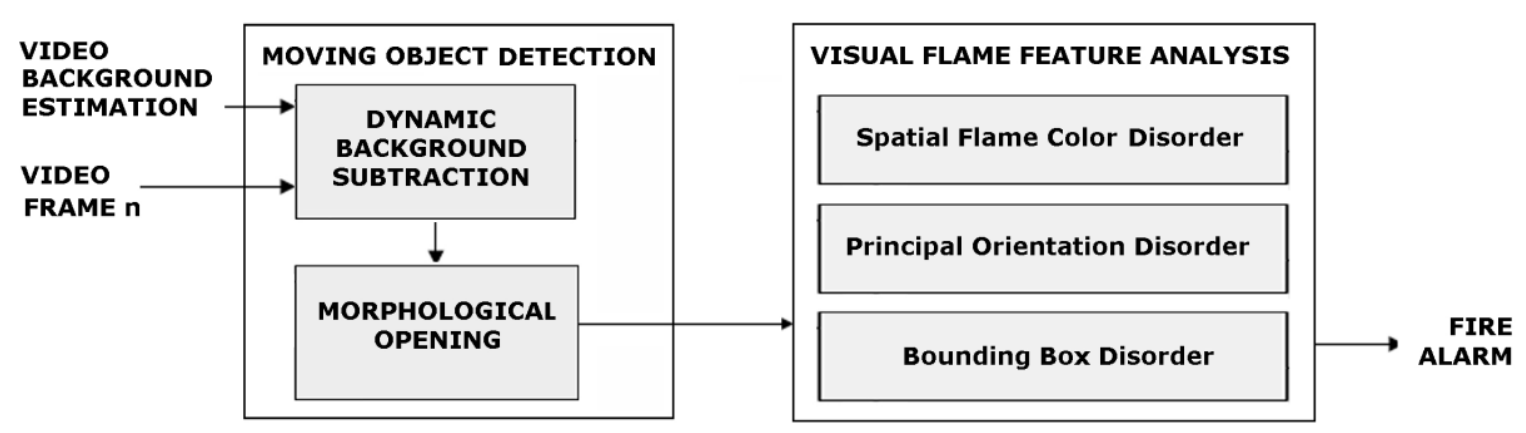

Fig. 1. Low-cost visual flame detector [12].

\section{LWIR-Based VFD}

Due to the fact that IR imaging is heading in the direction of higher resolution, increased sensitivity and higher speed, it is already used successfully as an alternative for ordinary video in many video surveillance applications, e.g., traffic safety and airport security. As manufacturers ensure price-reduction, it is even expected that this number of IR imaging applications will increase significantly in the near future $[13,14]$.

Although the trend towards IR-based video analysis is noticeable, the number of papers about IR-based fire detection is still limited. As is, this relatively new subject in vision research has still a long way to go. Nevertheless, the results from existing work already seem very promising and ensure the feasibility of IR video in fire detection. Owrutsky et al. [15] work in the near infrared (NIR) spectral range and compare the global luminosity $L$, which is the sum of the pixel intensities of the current frame, to a reference luminosity $L_{b}$ and a threshold $L_{t h}$. If the number of consecutive frames where $L>L_{b}+L_{t h}$ exceeds a persistence criterion, the system goes into alarm. Although this fairly simple algorithm seems to produce good results in the reported experiments its limited constraints do raise questions about its applicability in large and open uncontrolled public places. Toreyin et al. [16] detect flames in infrared by searching for brightlooking moving objects with rapid time-varying contours. A wavelet domain analysis of the 1D-curve representation of the contours is used to detect the high frequency nature of the boundary of a fire region. In addition, the temporal behavior of the region is analyzed using a Hidden Markov Model (HMM). The combination of both temporal and spatial clues seems more appropriate than the luminosity approach and, according to Toreyin et al., greatly reduces false alarms caused by ordinary bright moving objects.

A similar combination of temporal and spatial features is also used by Bosch et al. [17]. Hotspots, i.e., candidate flame regions, are detected by automatic histogram-based image thresholding. By analyzing the intensity, signature, and orientation of these resulting hot objects' regions, discrimination between flames and other objects is made. The IR-based fire detector (Fig. 2), proposed by the authors in Ref. [18], mainly follows the latter feature-based strategy, but contrary to Bosch et al.'s work a dynamic background subtraction method is used which is more suitable to cope with the time-varying characteristics of dynamic scenes. Also, by changing the set of features and combining their probabilities into a global classifier, a decrease in computational complexity and execution time is achieved with no negative effect on the detection results. 


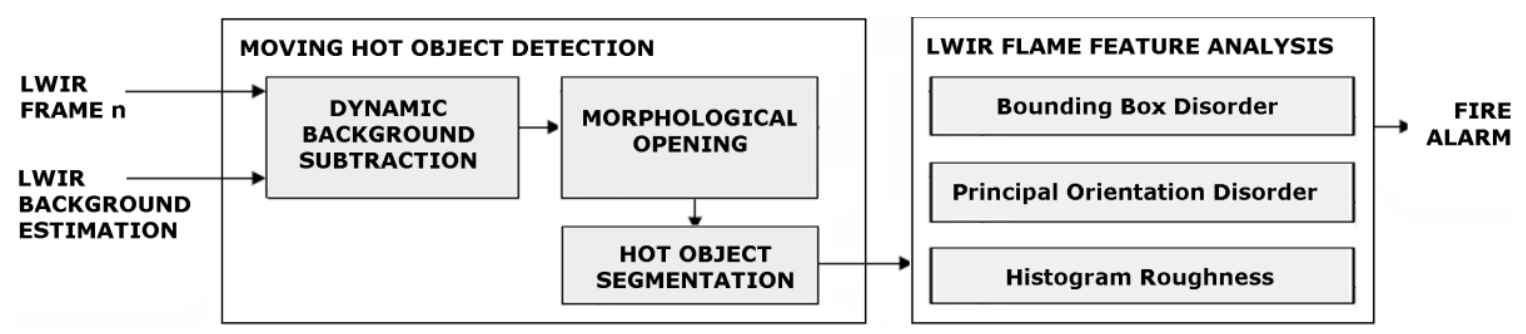

Fig. 2. Low-cost LWIR flame detector [18].

Similar to the visual flame detector, the LWIR detector starts with a dynamic background subtraction (Fig. 3a-c) and morphological filtering. Then, it automatically extracts hot objects (Fig. 3d) from the foreground thermal images by histogram-based segmentation, which is based on Otsu's method [19].

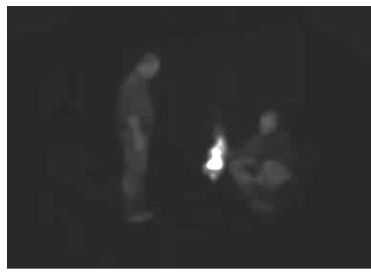

a) LWIR-VIDEO frame $n$

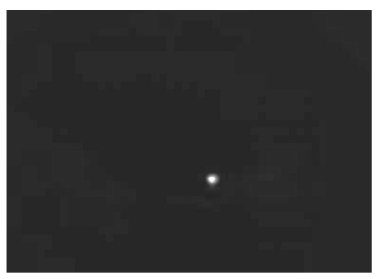

b) BACKGROUND ESTIMATION

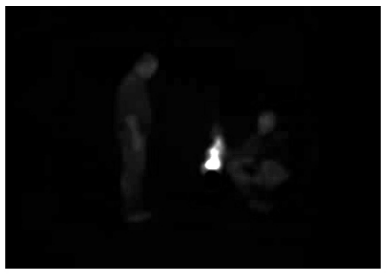

c) BACKGROUND SUBTRACTION

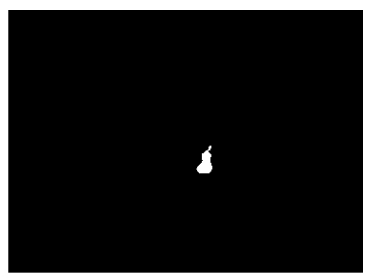

d) HOT OBJECT SEGMENTATION

Fig. 3. Thermal filtering: moving hot object segmentation.

After this thermal filtering, only the relevant hot objects in the scene remain in the foreground. These objects are then further analyzed using a set of three LWIR fire features: bounding box disorder, principal orientation disorder, and histogram roughness. The set of features is based on the distinctive geometric, temporal and spatial disorder characteristics of bright flame regions, which are easily detectable in LWIR thermal images. By combining the probabilities of these fast retrievable local flame features we are able to detect the fire at an early stage. Experiments with different LWIR fire/non-fire sequences show already good results, as indicated in (Table 1) by the flame detection rate, i.e. the percentage of correctly detected fire frames. These experiments were evaluated using manually annotated ground truth (GT).

Table 1. Experimental results of LWIR-based video fire detection.

\begin{tabular}{|l|c|c|c|c|c|c|}
\hline Video sequence & $\begin{array}{c}\text { No. } \\
\text { frames }\end{array}$ & $\begin{array}{c}\text { No. fire } \\
\text { frames } \\
\text { (GT) }\end{array}$ & $\begin{array}{c}\text { No. } \\
\text { detected } \\
\text { fire frames }\end{array}$ & $\begin{array}{c}\text { Mean } \\
\text { P(flames) }\end{array}$ & $\begin{array}{c}\text { No. false } \\
\text { detections }\end{array}$ & $\begin{array}{c}\text { Flame } \\
\text { detection } \\
\text { rate }^{\text {a }}\end{array}$ \\
\hline Attic (fire) & 337 & 264 & 255 & 0.91 & 9 & 0.93 \\
\hline $\begin{array}{l}\text { Attic (fire + moving } \\
\text { people) }\end{array}$ & 2123 & 1461 & 1296 & 0.86 & 34 & 0.86 \\
\hline Attic (moving people) & 886 & 0 & 14 & 0.24 & 14 & - \\
\hline Lab (Bunsen burner) & 115 & 98 & 77 & 0.83 & 0 & 0.79 \\
\hline $\begin{array}{l}\text { Corridor (person + hot } \\
\text { object) }\end{array}$ & 184 & 0 & 8 & 0.29 & 8 & - \\
\hline
\end{tabular}

${ }^{\mathrm{a}}$ detection rate $=($ no. detected fire frames - no. false detections $) /$ no. fire frames

\section{MULTI-SENSOR SMOKE AND FIRE DETECTION}

Recently, the fusion of visible and infrared images is starting to be explored as a way to improve detection performance in video surveillance applications. The combination of both types of imagery yields information about the scene that is rich in color, motion and thermal detail. Once the images are registered, i.e. aligned with each other, such information can be used to successfully detect and analyze activity in the scene. To detect fire, one can also take advantage of this multi-sensor benefit. 
The proposed multi-sensor flame and smoke detection can be split up into two consecutive parts: the registration of the multi-modal images and the detection itself. In the following subsections each of these parts will be discussed more in detail.

\section{Image Registration}

The image registration process (Fig. 4) detects the geometric parameters which are needed to overlay images of the same scene taken by different sensors. The registration starts with a moving object silhouette extraction [20] to separate the calibration objects, i.e. the moving foreground, from the static background. Key components are the dynamic background (BG) subtraction, automatic thresholding and morphological filtering. Then, 1-D contour vectors are generated from the resulting IR/visual silhouettes using silhouette boundary extraction, cartesian to polar transform and radial vector analysis. Next, to retrieve the rotation angle ( contour alignment) and the scale factor between the LWIR and visual image, the contours are mapped onto each other using circular cross correlation [21] and contour scaling. Finally, the translation between the two images is calculated using maximization of binary correlation. The retrieved geometric parameters are used in the second part of the multi-sensor detectors to align the visual and thermal images.

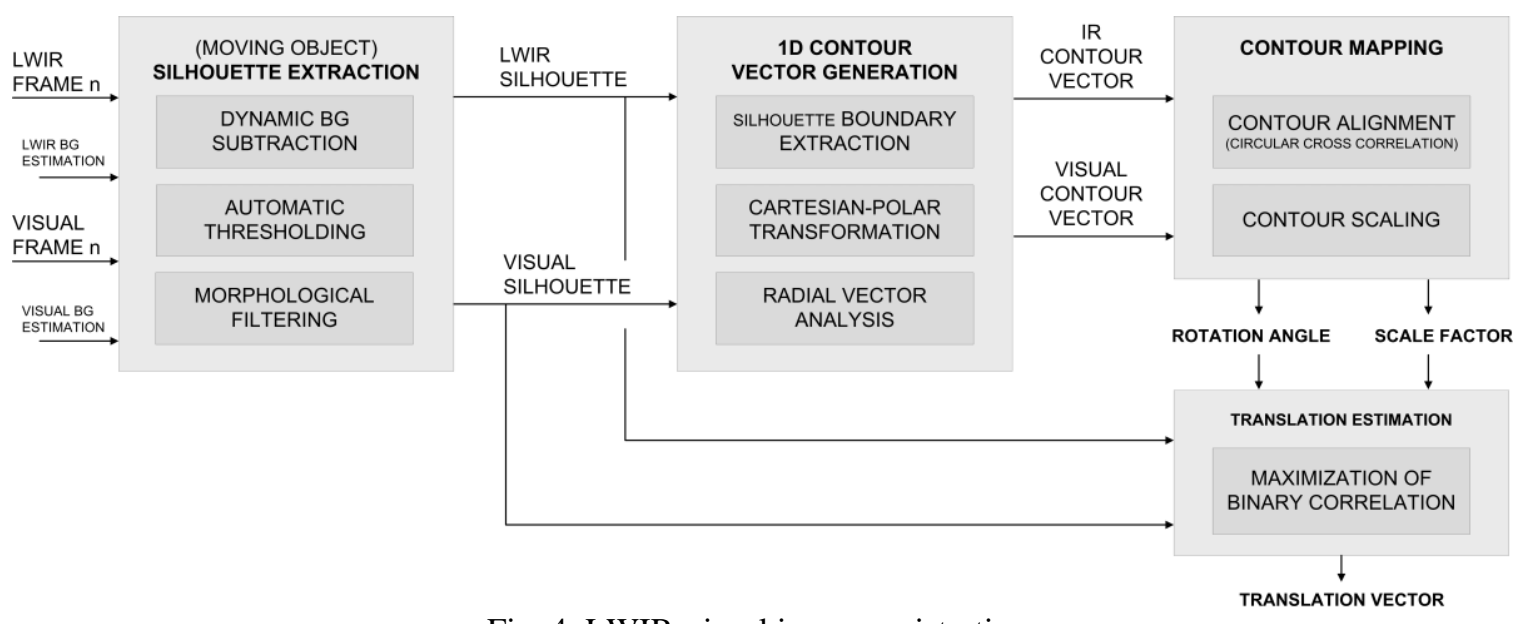

Fig. 4. LWIR-visual image registration.

\section{Multi-Sensor Flame Detection}

The multi-sensor flame detection (Fig. 5) first searches for candidate flame objects in both LWIR and visual images by using moving object detection and flame feature analysis. These steps have already been discussed previously. Next, it uses the registration information, i.e. rotation angle, scale factor and translation vector, to map the LWIR and visual candidate flame objects on each other. Finally, the global classifier analyzes the probabilities of the mapped objects. In case objects are detected with a high combined multi-sensor probability, a fire alarm is given.

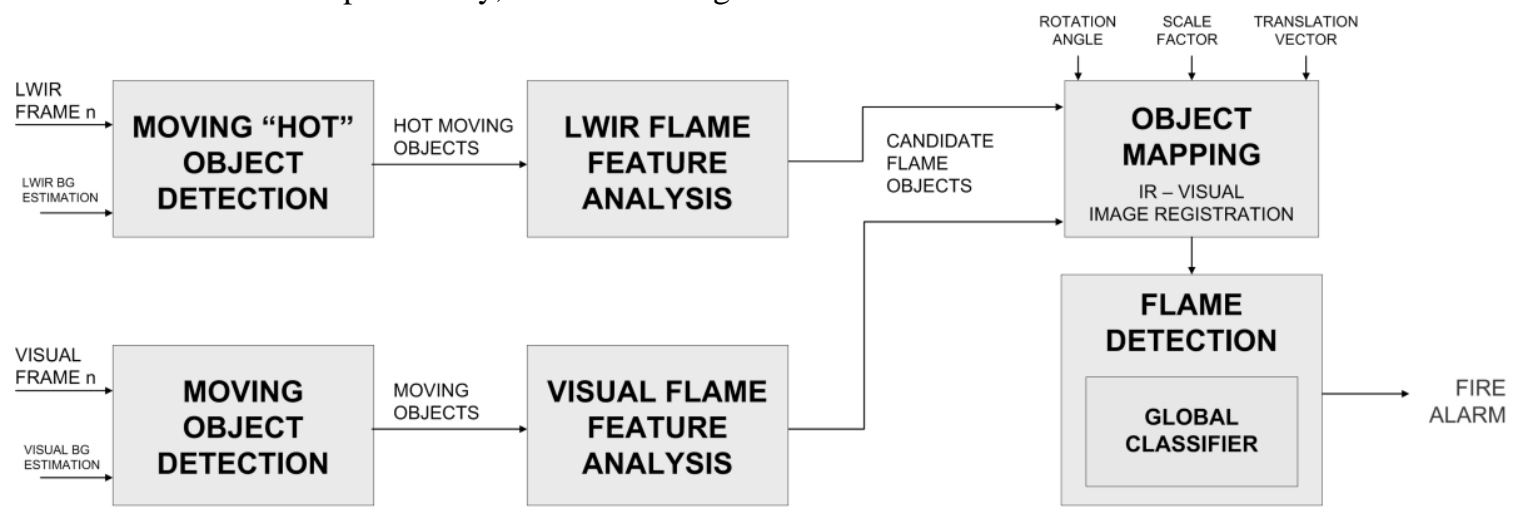

Fig. 5. Multi-sensor flame detection. 
As can be seen in (Table 2), the multi-sensor flame detector yields better results than the LWIR detector alone (Table 1). In particular for uncontrolled fires, a higher flame detection rate with fewer false alarms is achieved. Compared to the rather limited results of standalone visual flame detectors [1], the multi-sensor detection results are also more positive. As such, the combined detector is a win-win. As the images of the experiments (Fig. 6) show, only objects which are detected as fire by both sensors do raise the fire alarm.

Table 2. Experimental results of multi-sensor video fire detection.

\begin{tabular}{|l|c|c|c|c|c|c|}
\hline Video sequence & $\begin{array}{c}\text { No. } \\
\text { frames }\end{array}$ & $\begin{array}{c}\text { No. fire } \\
\text { frames } \\
\text { (GT) }\end{array}$ & $\begin{array}{c}\text { No. } \\
\text { detected } \\
\text { fire frames }\end{array}$ & $\begin{array}{c}\text { Mean } \\
\text { P(flames) }\end{array}$ & $\begin{array}{c}\text { No. false } \\
\text { detections }\end{array}$ & $\begin{array}{c}\text { Flame } \\
\text { detection } \\
\text { rate }^{\mathrm{a}}\end{array}$ \\
\hline Attic (fire) & 337 & 264 & 259 & 0.92 & 6 & 0.96 \\
\hline $\begin{array}{l}\text { Attic (fire + moving } \\
\text { people) }\end{array}$ & 2123 & 1461 & 1352 & 0.84 & 19 & 0.91 \\
\hline Attic (moving people) & 886 & 0 & 5 & 0.22 & 5 & - \\
\hline Lab (Bunsen burner) & 115 & 98 & 74 & 0.77 & 0 & 0.75 \\
\hline $\begin{array}{l}\text { Corridor (person + hot } \\
\text { object) }\end{array}$ & 184 & 0 & 3 & 0.28 & 3 & - \\
\hline
\end{tabular}

${ }^{a}$ detection rate $=$ (no. detected fire frames - no. false detections $) /$ no. fire frames
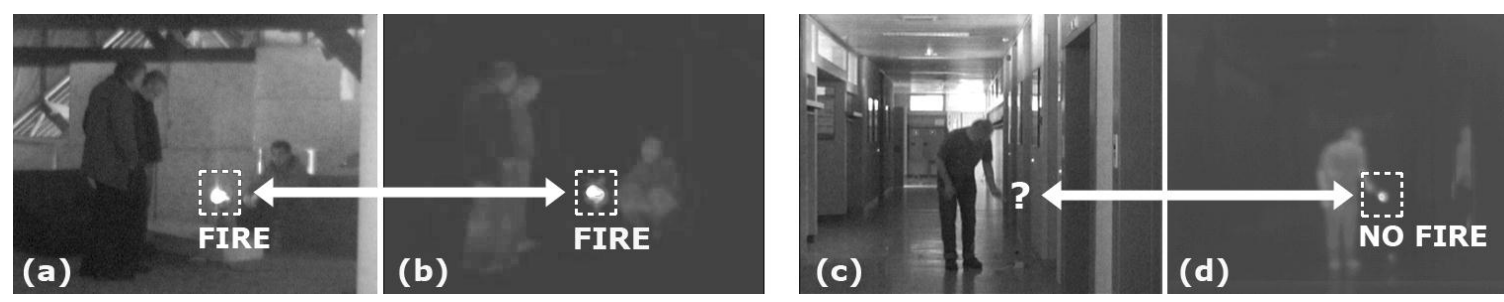

Fig. 6. LWIR fire detection experiments.

\section{Multi-Sensor Smoke Detection}

The proposed multi-sensor smoke detector makes use of the invisibility of smoke in LWIR. Smoke, contrarily to ordinary moving objects, will only be detected in visual images. As such, the coverage of moving objects their LWIR and visual silhouettes will start to decrease in case of smoke. Due to the dynamic character of the smoke, this decrease will also show a high degree of disorder. By focusing on both coverage behaviors, the system is able to accurately detect the smoke.

The silhouette coverage analysis (Fig. 7) starts with the similar moving object silhouette extraction as the one used for registration. Then, it uses the registration information, i.e. rotation angle, scale factor and translation vector, to map the IR and visual silhouette images on each other. Finally, the coverage of the resulting IR-visual silhouette map is computed and is analyzed over time. In case of silhouette coverage reduction with a high degree of disorder, fire alarm is given.

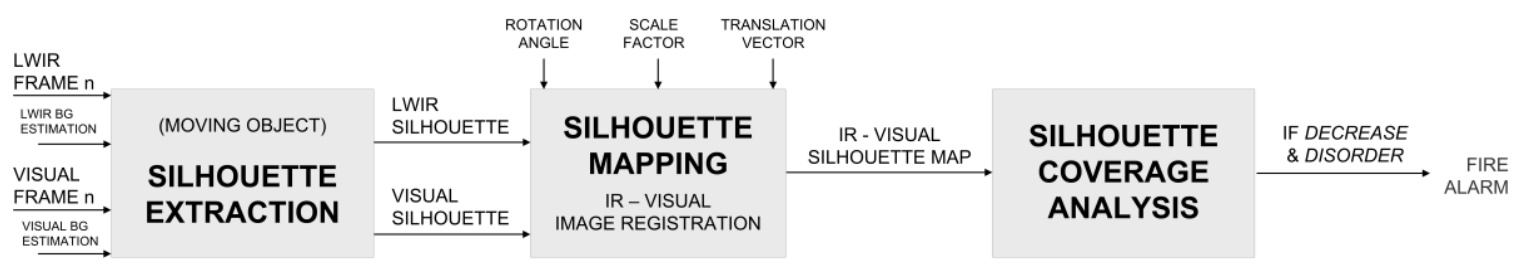

Fig. 7. Multi-sensor smoke detection. 
The silhouette maps in Fig. 8 show that the proposed approach achieves good performance for image registration between color and thermal image sequences. The visual and IR silhouette of the person are coarsely mapped on each other. Due to the individual sensor limitations, such as shadows in visual images, thermal reflections and soft thermal boundaries in LWIR, small artifacts at the boundary of the merged silhouettes can be noticed. This is also the reason why the LWIR-visual silhouette coverage for ordinary moving objects is between 0.7 and 0.8 , and not equal to 1 . As can be seen in the silhouette coverage graph of the moving person, this $0.7-0.8$ coverage remains quasi-constant over time. Contrarily, for the smoke sequence the silhouette coverage decreases as soon as smoke occurs and shows a high degree of disorder. Using quantization and extrema analysis these phenomena are detected and a fire alarm is raised. Even in the case where no moving objects are present, the system detects the smoke.

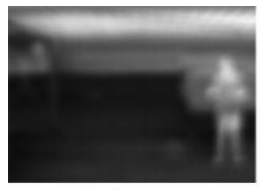

IR frame $n$

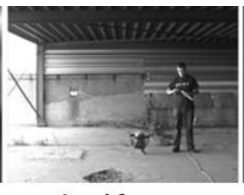

visual frame $n$

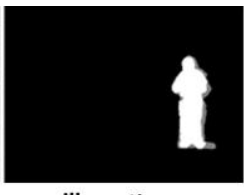

silhouette map

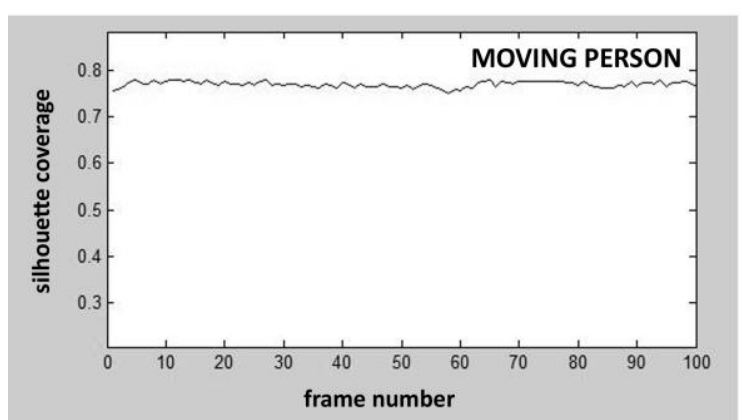

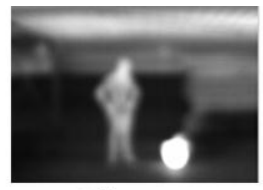

IR frame $n$

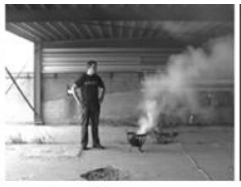

visual frame $n$

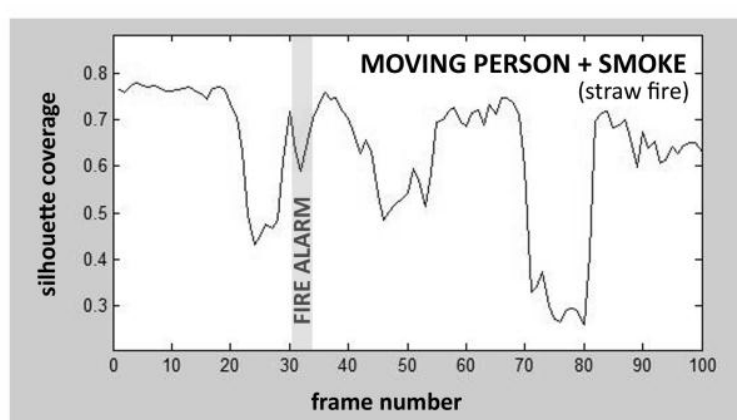

Fig. 8. Experimental results: silhouette coverage analysis.

\section{MULTI-VIEW FIRE ANALYSIS}

Only a few of the existing VFD systems [22,23] are capable of providing additional information on the fire circumstances, such as size and location. Despite the good performance reported in the papers, the results of these approaches are still limited and interpretation of the provided information is not straightforward. As such, one of the main goals of our work is to provide an easy-to-use and information-rich framework for video fire analysis, which is discussed briefly in this paper. For more details, readers are referred to [2].

Using the localization framework shown in Fig. 9, information about the fire location and (growing) size can be generated very accurately. First, the framework detects the fire, i.e. smoke and/or flames, in each single view. An appropriate single-view smoke or flame detector can be chosen out of the numerous approaches already proposed previously. It is even possible to use the multi-sensor detectors. The only constraint is that the detector produces a binary image as output, in which white regions are fire/smoke FG regions and black regions are non-fire/non-smoke BG. Secondly, the single-view detection results of the available cameras are projected by homography [24] onto horizontal and vertical planes which slice the scene. For optimal performance it is assumed that the camera views overlap. Overlapping multi-camera views provide elements of redundancy, i.e., each point is seen by multiple cameras that help to minimize ambiguities like occlusions, i.e. visual obstructions, and improve the accuracy in the determination of the position and size of the flames and smoke. Next, the plane slicing algorithm accumulates, i.e. sums, the multi-view detection results in each of the horizontal and vertical planes. This step is a 3-D extension of Arsic's work [25]. Then, a 3-D grid of virtual multi-camera sensors is created at the crossings of these planes. At each sensor point of the grid, the detection results of the horizontal and vertical planes that cross at that point are analyzed and only the points with stable detections are further considered as candidate fire or smoke. Finally, 3-D spatial and temporal filters clean up the grid and remove the remaining noise. The filtered grid can then be used to extract the smoke and fire location, information about the growing process and the direction of propagation. For a more detailed description the reader is referred to Ref. [26]. 


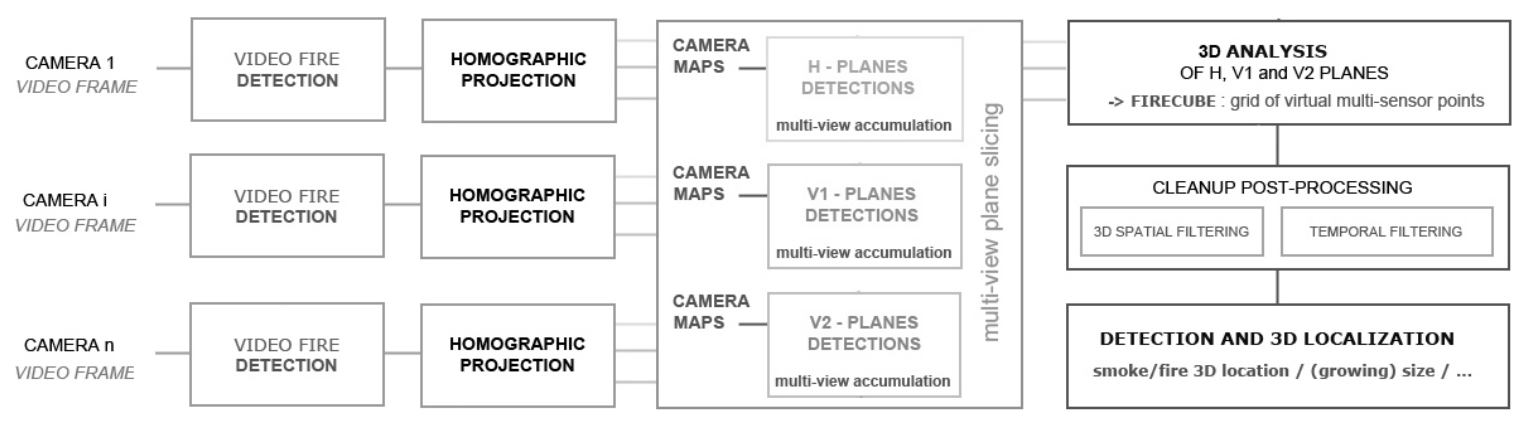

Fig. 9. Multi-view localization framework for 3-D fire analysis [2].

Since fire/smoke regions are not always visible in all views or only partly visible, the detection/localization will be influenced by the number of cameras having the fire/smoke position under surveillance. By making a camera map, that contains the number of cameras that are able to monitor the specific position, appropriate detection criteria can be determined. The higher the value of a position on the map, the more cameras that monitor that position. Special events, such as camera unavailability and tampering can be automatically detected and could also be taken into account by dynamically updating the map [26].

In order to verify the proposed multi-view localization framework we performed smoke experiments in a car park. We tried to detect the location, the growing size and the propagation direction of smoke generated by a smoke machine. An example of these experiments is shown in Fig. 10. Single-view fire detection results, i.e. the binary images which are the input for the homographic projection in our localization framework, were retrieved by using the chrominance-based smoke detection method proposed in Ref. [1]. Since the framework is independent of the type of VFD, also other detectors can be used here. The only constraint is that the detector delivers a black and white binary image, as mentioned earlier. As such, it is even possible to integrate other types of sensors, such as the proposed multi-sensor detectors.
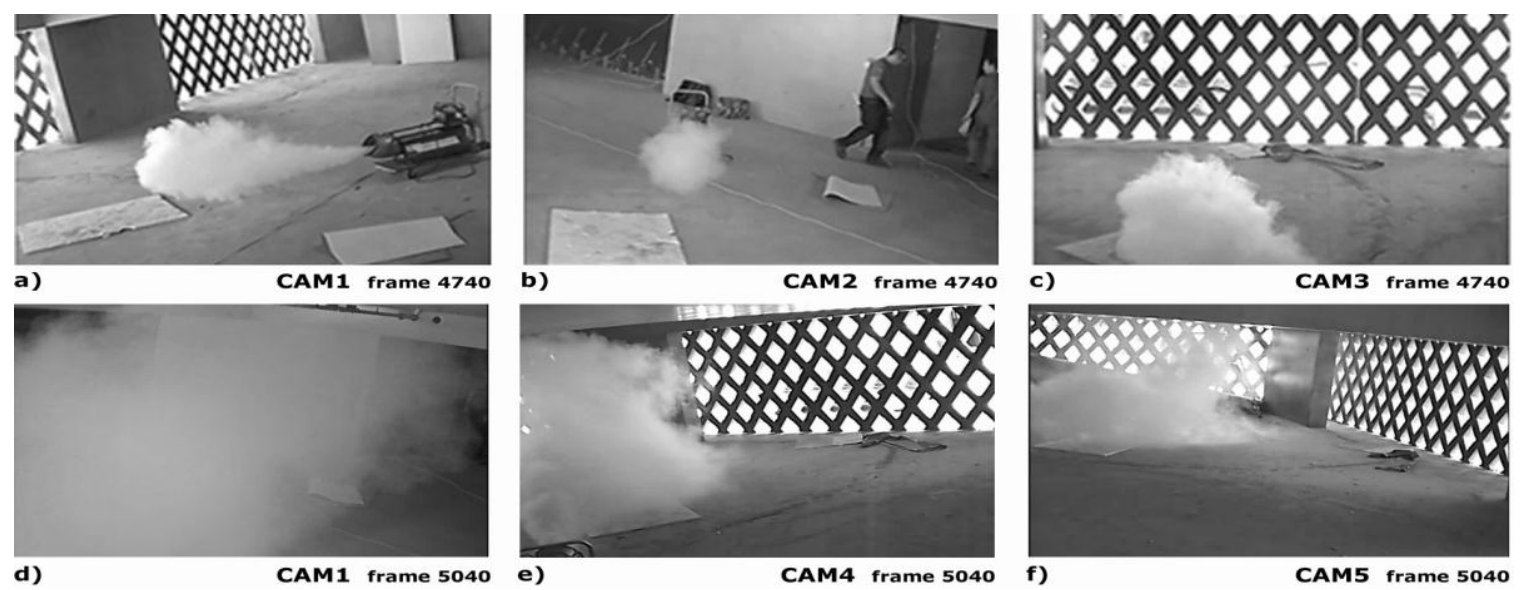

d)

CAM1 frame 5040 e)

CAM4 frame 5040

CAM5 frame 5040

Fig. 10. Car park smoke experiments.

As can be seen in the 3-D model in Fig. 11 and in the back-projections of the 3-D results in Fig. 12, the framework is able to detect the location and the dimension of the smoke regions. In Fig. 11 the smoke regions are represented by the dark gray 3-D boxes, which are bounded by the minimal and maximal horizontal and vertical FG slices. As a reference, also the bounding box of the smoking machine is visualized. Even if a camera view is partially or fully occluded by smoke, like for example in frame 5040 of CAM2 (Fig. 10d), the framework localizes the smoke, as long as it is visible from the other views. Based on the detected 3-D smoke boxes, the framework generates the spatial smoke characteristics, i.e., the height, width, length, centroid, and volume of the smoke region. By analyzing this information over time, the growing size and the propagation direction are also estimated. If LWIR-visual multi-sensor cameras, like the one proposed in this paper, are used, it is even possible to also analyze the temperature evolution of the detected regions. As such, for example, temperature-based levels of warnings can be given. 


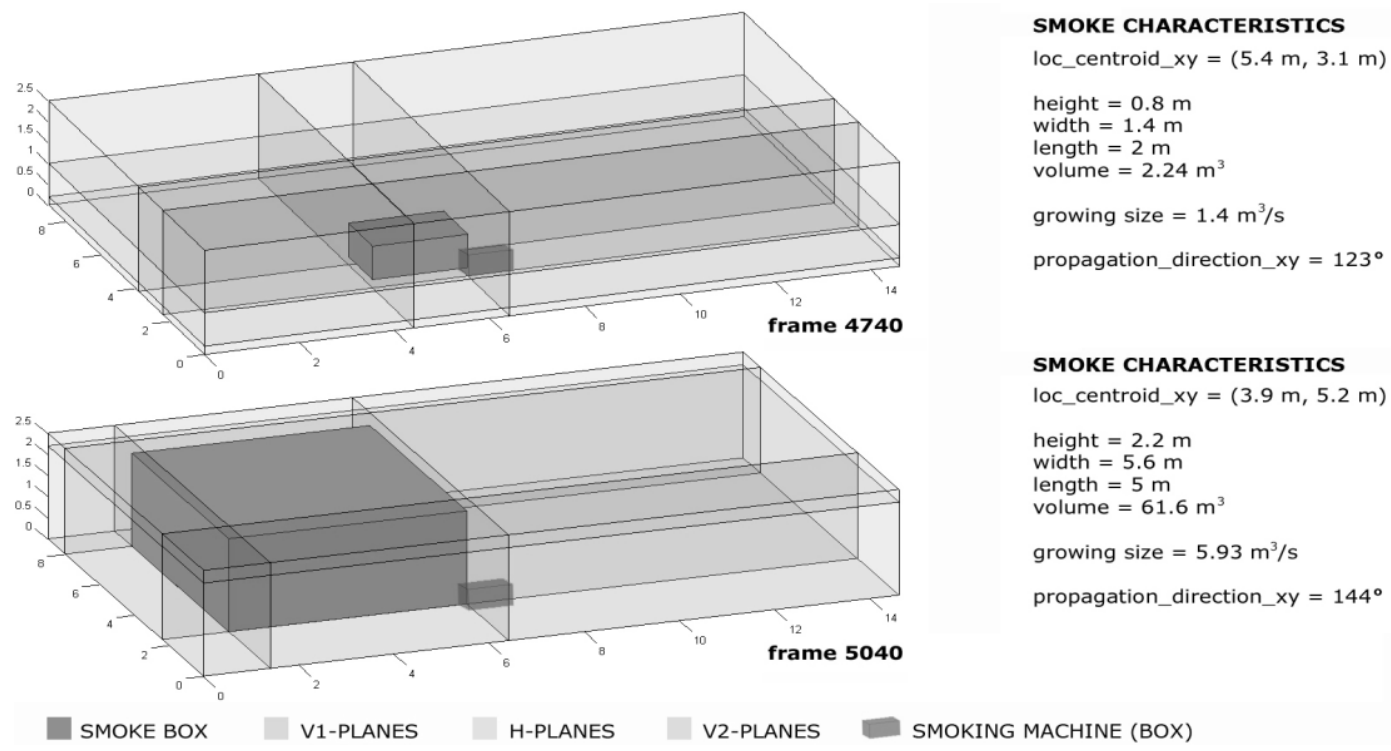

Fig. 11. Plane slicing-based smoke box localization.

The back-projections (Fig. 12) of the 3-D smoke regions to the camera views show that the multi-view slicing approach produces plausible and acceptable results. Due to the fact that the number of (multi-view) video fire sequences is limited and the fact that no 3-D ground truth data and widely "agree-upon" evaluation criteria of video-based fire tests are available yet [27], only this kind of visual validation is possible for the moment. Contrary to existing fire analysis approaches [23], which deliver a rather limited 3 -D reconstruction, the output contains valuable 3-D information about the fire development.
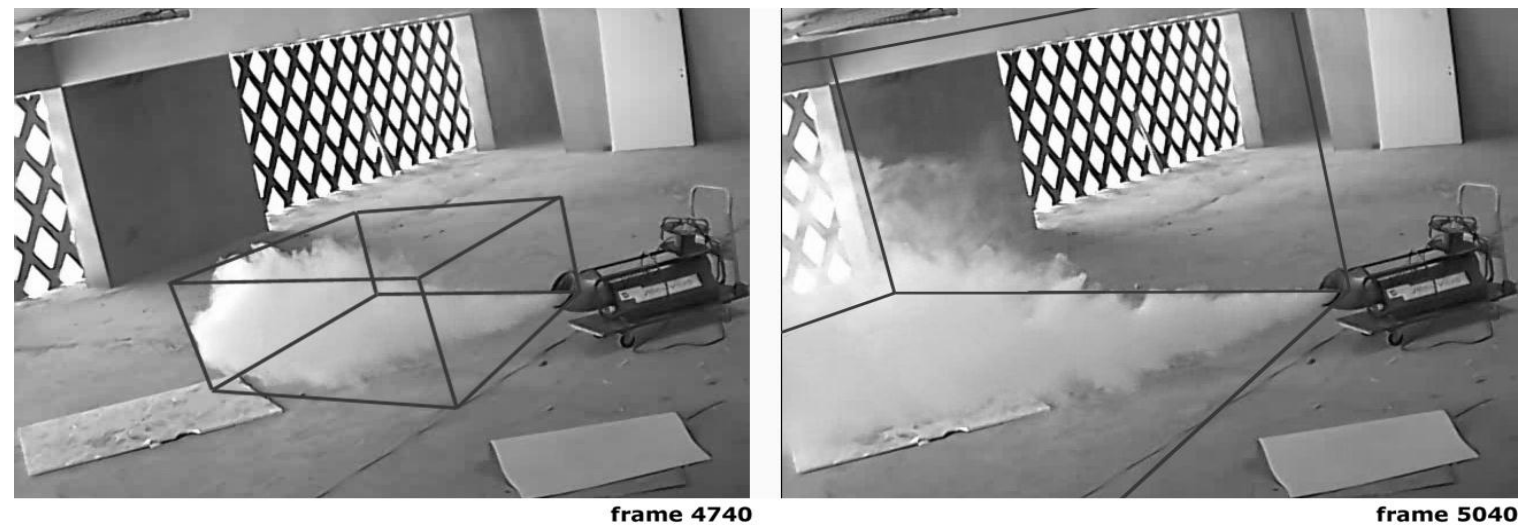

Fig. 12. Back-projection of 3-D smoke box results into camera view CAM1.

\section{FUTURE DIRECTIONS}

\section{VFD Performance Evaluation}

Due to the limited number of fire datasets, the absence of ground truth (GT) data, the extensive use of heuristic thresholds and the lack of standardized evaluation criteria and metrics, experimental verification of VFD algorithms is still an error-prone and time-consuming task. To facilitate the evaluation process, and to provide a tool to correctly validate the effectiveness of video-based detectors in a standardized way, the authors propose a performance evaluation framework in Ref. [28].

The evaluation framework is able to determine optimal settings for each individual VFD algorithm and is also able to compare multiple algorithms against each other. By using ground truth data of a large set of fire and non-fire video sequences and comparing the detection results of each algorithm against this data, the 
framework ensures a reliable independent evaluation. Key components to perform this evaluation are the XML-based GT creation, an automatic VFD optimizer and frame and object-based evaluation metrics.

A general overview of the evaluation framework is shown in Fig. 13. The framework is designed for implementing new detection algorithms that still need to be tested for their optimal settings. This is done by the automatic video algorithm optimizer, which allows a detection algorithm to define a set of parameters that can be set for minimum and maximum values. These values are then incrementally run through on a set of fire and non-fire video files. Afterwards, the result per parameter combination is written to an XML file. The evaluation framework also contains an application that supports the creation of GT data for a given video. Because this GT creation is done manually by a user, it is still in some way error prone. As such, research on automatic GT annotation is part of our future work. Finally, the framework also includes a tool for comparing the GT data with the results created by the algorithm tests. This result set evaluation tool uses a variety of evaluation metrics $[28,29]$ to decide what detection algorithm performs best and outputs the optimal parameters for a given set of videos.

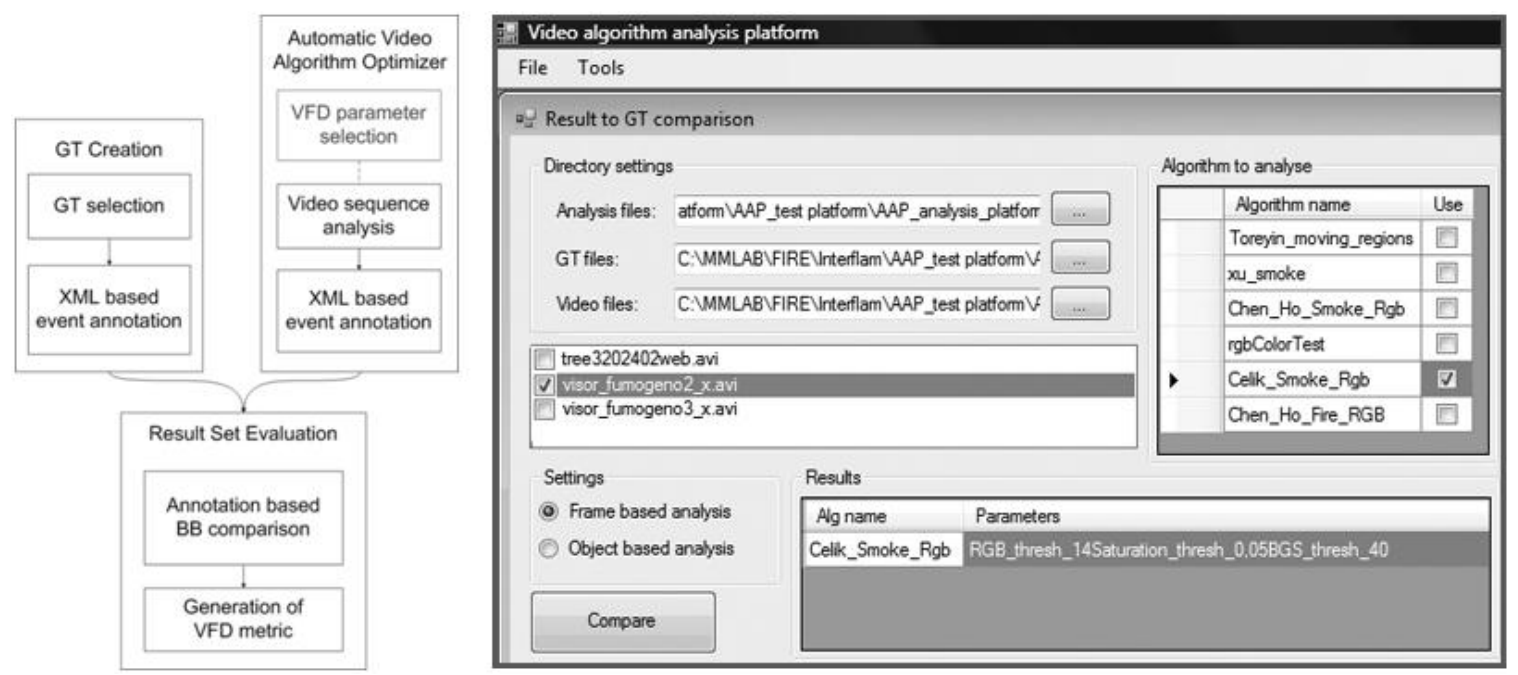

Fig. 13. VFD performance evaluation framework.

Prior experiments [28] indicate that the VFD performance evaluation framework is very promising and is a worthy alternative for the error-prone and time-consuming experimental evaluations that are used in many works today. However, further testing on a broader range of fire and non-fire video sequences is necessary for an adequate evaluation. As future work, we will also look for ways to remove the human factor in the GT creation process. This would relieve the research teams as they would only need to research a detection algorithm, select what type of object(s) the algorithm must detect and let the system create a test bench.

\section{Video-Driven Fire Spread Forecasting}

Fire spread forecasting is about predicting the further evolution of a fire, in the event of the fire itself. In the world of fire research, not much experience exists on this topic [30]. Based on their common use in fire modeling, CFD (computational fluid dynamics) [31] calculations look interesting for fire forecasting at first sight. These are three-dimensional simulations where the rooms of interest are subdivided into a large amount of small cells (Fig. 14b). In each cell, the basic laws of fluid dynamics and thermodynamics (conservation of mass, total momentum and energy) are evaluated in time. These types of calculations result in quite accurate and detailed results, but they are costly, especially in calculation time. As such, CFD simulations do not seem to be the most suitable technique for fast fire forecasting. We believe, therefore, it is better to use zone models [31] to perform this task.

In a zone model, the environment is subdivided into two main zones. The smoke of the fire is in the hot zone. A cold air layer exists underneath this hot zone (Fig. 14a). The interface between these two zones is an essentially horizontal surface. The height of the interface $\left(h_{\text {int }}\right)$ and the temperature of the hot $\left(T_{h o t}\right)$ and cold $\left(T_{\text {cold }}\right)$ zones vary as function of time. These calculations are simple in nature. They rely on a set of 
experimentally derived equations for fire and smoke plumes. It usually takes between seconds and minutes to perform this kind of calculations, depending on the simulated time and the dimensions of the room or building. Therefore, it is much better suited for fire forecasting than the use of CFD calculations.

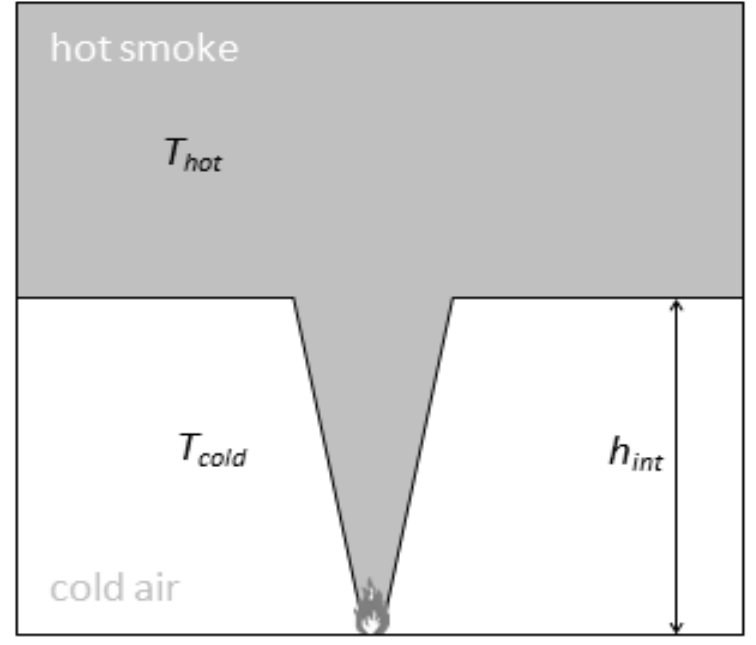

(a)

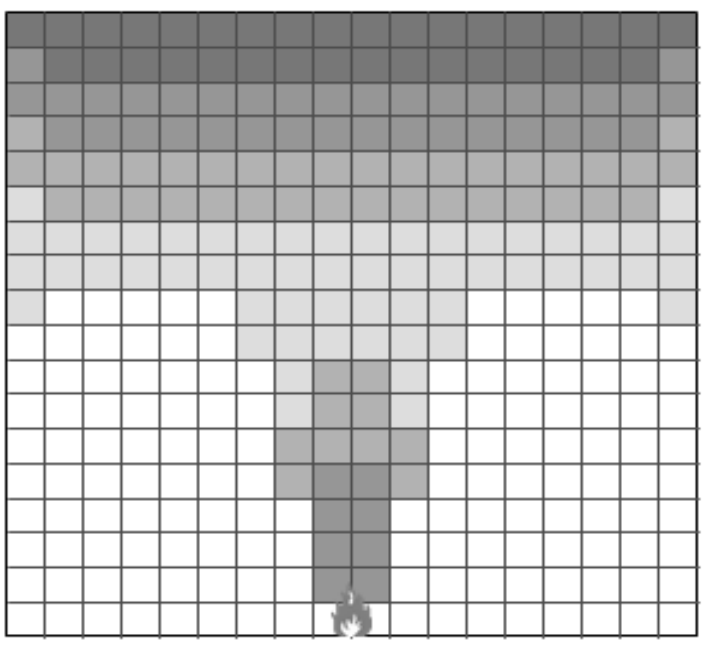

(b)

Fig. 14. Fire modeling techniques: (a) zone model; (b) computational fluid dynamics (CFD) model.

The real aim of fire forecasting is to use measured data from the fire, e.g. obtained by sensors or video images in the room of interest, in order to replace or correct the model predictions [32,33]. This process of data assimilation is illustrated in Fig. 15, which summarizes our future plans for video-driven fire forecasting. As can be seen in the graph, model predictions of smoke layer height $(\sim$ zone model interface $h_{\text {int }}$ ) are corrected at each correction point. This correction uses the measured smoke characteristics from our fire analysis framework. The further we go in time, the closer the model begins to match the future measurements and the more accurate predictions of future smoke layer height become.

The proposed video-driven fire forecasting is a prime example of how video-based detectors will be able to do more than just generate alarms. Detectors can give information about the state of the environment, and using this information, zone model-based predictions of the future state can be improved and accelerated. By combining the information about the fire from models and real-time data we will be able to produce an estimate of the fire that is better than could be obtained from using the model or the data alone.

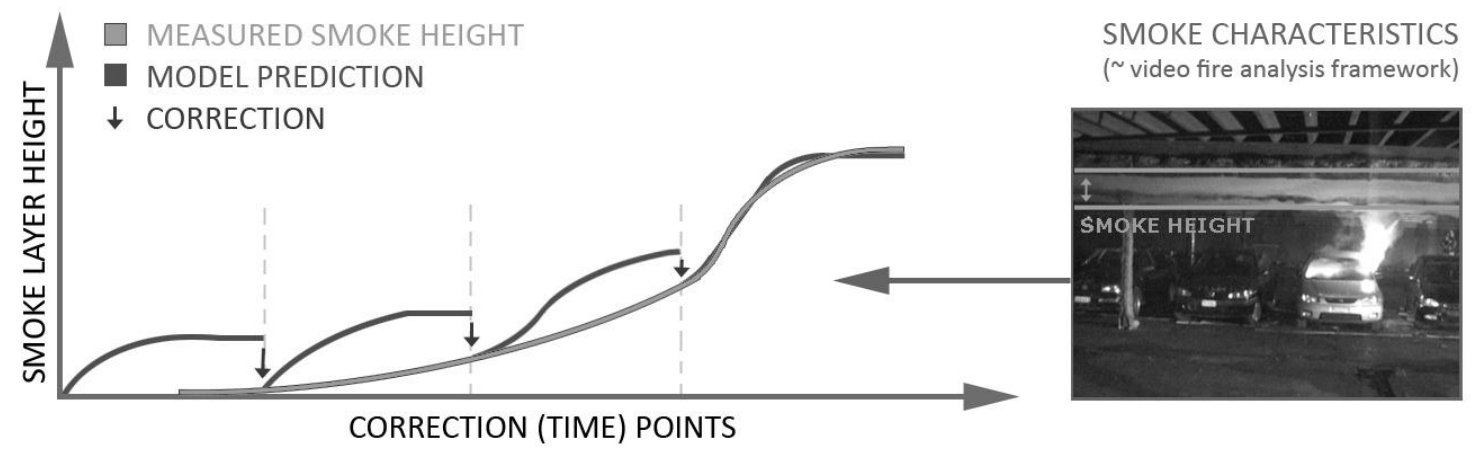

Fig. 15. Data assimilation - video-driven fire forecasting (FireGrid [32]). 


\section{CONCLUSIONS}

Based on the analysis of existing approaches in visible and non-visible light and on our own experiments, this paper presents a multi-sensor fire detector which detects flames and smoke in LWIR and visual registered images. By using thermal and visual images to detect and recognize the fire, we can take advantage of the different kinds of information to improve the detection and to reduce the false alarm rate. To detect the presence of flames at an early stage, the novel multi-sensor flame detector fuses visual and non-visual flame features from moving (hot) objects in ordinary video and LWIR thermal images. By focusing on the distinctive geometric, temporal and spatial disorder characteristics of flame regions, the combined features are able to successfully detect flames. The novel multi-sensor smoke detector, on the other hand, makes use of the smoke invisibility in LWIR. The smoke detector analyzes the silhouette coverage of moving objects in visual and LWIR registered images. In case of silhouette coverage reduction with a high degree of disorder, a fire alarm is given. Experiments on both fire and non-fire multi-sensor sequences indicate that the proposed algorithm can detect the presence of smoke and flames in most cases. Moreover, false alarms, one of the major problems of many other VFD techniques, are drastically reduced. As a future work, additional 'evaluation' experiments will also be performed following the guidelines from the work of Gottuk and Dinaburg [34].

To provide more valuable information about the fire progress, we also present a multi-view fire analysis framework, which is mainly based on 3-D extensions to homographic plane slicing. The framework merges single view VFD results of multiple cameras by homographic projection onto multiple horizontal and vertical planes which slice the scene under surveillance. At the crossings of these slices, we create a 3-D grid of virtual sensor points. Using this grid, information about 3-D location, size and propagation of the fire can be extracted from the video data. As prior experimental results show, this combined analysis from different viewpoints provides more valuable fire characteristics.

\section{ACKNOWLEDGEMENTS}

The research activities as described in this paper were funded by Ghent University, the Interdisciplinary Institute for Broadband Technology (IBBT), University College West Flanders, WarringtonFireGent, Xenics, the Institute for the Promotion of Innovation by Science and Technology in Flanders (IWT), the Fund for Scientific Research-Flanders (FWO-Flanders G.0060.09), the Belgian Federal Science Policy Office (BFSPO), and the European Union.

\section{REFERENCES}

[1] Verstockt, S., Merci, B., Sette, B., Lambert, P., and Van de Walle, R., "State of the art in visionbased fire and smoke detection," AUBE'09 - Proceedings of the 14th International Conference on Automatic Fire Detection, University of Duisburg-Essen, 2009, pp. 285-292.

[2] Verstockt, S., Van Hoecke, S., Tilley, N., Merci, B., Sette, B., Lambert, P., Hollemeersch, C., and Van de Walle, R, (2011) FireCube: a multi-view localization framework for 3D fire analysis, (accepted in) Fire Safety Journal.

[3] Celik, T. and Demirel, H., (2009) Fire detection in video sequences using a generic color model, Fire Safety Journal 44(2): 147-158, http://dx.doi.org/10.1016/j.firesaf.2008.05.005

[4] Chen, T.-H., Wu, P.-H., and Chiou, Y.-C., "An early fire-detection method based on image processing," International Conference on Image Processing, ICIP, 2004, pp. 1707-1710.

[5] Qi, X., and Ebert, J., (2009) A computer vision based method for fire detection in color videos, International Journal of Imaging 2: 22-34.

[6] Marbach, G., Loepfe, M., and Brupbacher, T., (2006) An image processing technique for fire detection in video images, Fire Safety Journal 41(4): 285-289, http://dx.doi.org/10.1016/j.firesaf.2006.02.001

[7] Calderara, S., Piccinini, P., and Cucchiara, R., "Smoke detection in video surveillance: a MoG model in the wavelet domain," International Conference on Computer Vision Systems, Springer Berlin / Heidelberg, 2008, pp. 119-128, http://dx.doi.org/10.1007/978-3-540-79547-6_12 
[8] Toreyin, "Contour based smoke detection in video using wavelets," European Signal Processing Conference, EUSIPCO, 2006, pp. 1-5.

[9] Töreyin, B., Dedeoğlu, Y., Güdükbay, U., and Çetin, A., (2006) Computer vision based method for real-time fire and flame detection, Pattern Recognition Letters 27(1): 49-58, http://dx.doi.org/10.1016/j.patrec.2005.06.015

[10] Borges, P. V. K., Mayer, J., and Izquierdo, E., "Efficient visual fire detection applied for video retrieval," $16^{\text {th }}$ European Signal Processing Conference, EUSIPCO, 2008, pp. 1-5.

[11] Xiong, Z., Caballero, R., Wang, H., Finn, A.M., Lelic, M.A., and Peng, P.-Y., "Video-based smoke detection: possibilities, techniques, and challenges," IFPA Fire Suppression and Detection Research and Applications-A Technical Working Conference, SUPDET, 2007, pp. 1-7.

[12] Verstockt, S., Vanoosthuyse, A., Van Hoecke, S., Lambert, P., and Van de Walle, R., "Multisensor fire detection by fusing visual and non-visual flame features," $4^{\text {th }}$ International Conference on Image and Signal Processing, Springer LNCS, 2010, pp. 333-341, http://dx.doi.org/10.1007/978-3-642-13681-8_39

[13] Vandersmissen, R., (2008) Night-vision camera combines thermal and low-light-level images, Photonik International 2: 2-4.

[14] Arrue, B., Ollero, A., and Matinez de Dios, J., (2000) An intelligent system for false alarm reduction in infrared forest-fire detection, IEEE Intelligent Systems 15(3): 64-73, http://dx.doi.org/10.1109/5254.846287

[15] Owrutsky, J., Steinhurst, D., Minor, C., Rosepehrsson, S., Williams, F., and Gottuk, D., (2006) Long wavelength video detection of fire in ship compartments, Fire Safety Journal 41(4): 315-320, http://dx.doi.org/10.1016/j.firesaf.2005.11.011

[16] Töreyin, B., Cinbiş, R., Dedeoğlu, Y., and Çetin, A., (2007) Fire detection in infrared video using wavelet analysis, Optical Engineering 46(6): 067204, http://dx.doi.org/10.1117/1.2748752

[17] Bosch, I., Gomez, S., Molina, and R., Miralles, "Object discrimination by infrared image processing," Proceedings of the $3^{\text {rd }}$ International Work-Conference on The Interplay Between Natural and Artificial Computation, Springer-Verlag Berlin, 2009, pp. 30-40, http://dx.doi.org/10.1007/978-3-642-02267-8_4

[18] Verstockt, S., Dekeerschieter, R., Vanoosthuise, A., Merci, B., Sette, B., Lambert, P., and Van de Walle, R., "Video fire detection using non-visible light," $\sigma^{\text {th }}$ International seminar on Fire and Explosion Hazards, ISFEH, 2010, pp. 1-12.

[19] Otsu, N., (1979) A threshold selection method from gray-level histograms, IEEE Transactions on Systems, Man, and Cybernetics 9: 62-66, http://dx.doi.org/10.1109/TSMC.1979.4310076

[20] Chen, H.-M., and Varshney, P.K., (2001) Automatic two-stage IR and MMW image registration algorithm for concealed weapons detection, IEE Proc. of Vision Image Signal Processing 148: 209-216, http://dx.doi.org/10.1049/ip-vis:20010459

[21] Hamici, Z., (2006) Real-time pattern recognition using circular cross-correlation: a robot vision system, International Journal of Robotics and Automation 21(3): 174-183, http://dx.doi.org/10.2316/Journal.206.2006.3.206-2724

[22] Yasmin, R., (2009) Detection of smoke propagation direction using color video sequences, Journal of Soft Computing 4: 45-48, http://dx.doi.org/10.3923/ijscomp.2009.45.48

[23] Akhloufi, M., Rossi, L., "Three-dimensional tracking for efficient fire fighting in complex situations," Visual Information Processing XVIII, SPIE, 2009, pp. 1-12, http://dx.doi.org/10.1117/12.818270

[24] Hartley, R., and Zisserman, A., Multiple view geometry in computer vision, Cambridge University Press, Cambridge, 2004, pp. 87-131. 
[25] Arsic, D., Hristov, E., Lehment, N., Hornler, B., Schuller, B., and Rigoll, G., "Applying multi layer homography for multi camera person tracking," Proceedings of the 2nd ACM/IEEE International Conference on Distributed Smart Cameras, ICDSC, 2008, pp. 1-9.

[26] Verstockt, S., Van Wallendael, G., De Bruyne, S., Poppe, C., Lambert, P., and Van de Walle, R., "Cube: a multi-view localization framework for 3D object analysis in computer vision applications," Computer Graphics International, CGI, 2010, pp. 1-4.

[27] Ko, B., Cheong, K., and Nam, J., (2009) Fire detection based on vision sensor and support vector machines, Fire Safety Journal 44(3): 322-329, http://dx.doi.org/10.1016/j.firesaf.2008.07.006

[28] Verstockt, S., Vanoosthuyse, A., Merci, B., Tilley, N., Sette, B., Hollemeersch, C., Lambert, P. and Van de Walle, R., "Performance evaluation framework for vision-based fire detection," $12^{\text {th }}$ International conference on Fire Science and Engineering (Interflam 2010), Interscience Communications, 2010, pp. 257-268.

[29] Baumann, A., Boltz, M., Ebling, J., Koenig, M., Loos, H., Merkel, M., Niem, W., Warzelhan, J., and Yu, J., (2008) A Review and Comparison of Measures for Automatic Video Surveillance Systems, EURASIP Journal on Image and Video Processing 2008: 1-30, http://dx.doi.org/10.1155/2008/824726

[30] Rein, G., Empis, C.A., and Carvel, R., The Dalmarnock Fire Tests: Experiments and Modelling, Engineering \& Electronics, University of Edinburgh, Edinburgh, 2007.

[31] Society of Fire Protection Engineers, The SFPE handbook of Fire Protection Engineering, National Fire Protection Association, Quincy, 2002.

[32] Welch, S., Usmani, A., Upadhyay, R., Berry, D., Potter, S., and Torero, J.L., "Introduction to FireGrid," The Dalmarnock Fire Tests: Experiments and Modelling, Engineering and Electronics, University of Edinburgh, Edinburgh, 2007.

[33] Jahn, W., Inverse Modelling to Forecast Enclosure Fire Dynamics, PhD Thesis, University of Edinburgh, Edinburgh, 2010.

[34] Gottuk, D.T., and Dinaburg, J.B., "Video image detection and optical flame detection for industrial applications," Fire suppression and detection research and applications - A technical working conference, SUPDET, 2010, pp. 1-32. 